\section{O22 3D PRINTED POLYETHYLENE OXIDE ORAL DOSES WITH INNOVATIVE 'RADIATOR-LIKE' DESIGN: IMPACT OF MOLECULAR WEIGHT ON MECHANICAL AND RHEOLOGICAL PROPERTIES AND DRUG RELEASE}

\begin{abstract}
1,2M Peak* ${ }^{*}{ }^{3} \mathrm{~K}$ Baj, ${ }^{4} \mathrm{~A}$ Isreb, ${ }^{5} \mathrm{M}$ Wojsz, ${ }^{6}$ Mohammad, ${ }^{7} \mathrm{M}$ Albed Alhnan. ${ }^{1}$ Paediatric Medicines Research Unit, Alder Hey Children's NHS Foundation Trust, Liverpool; ' University of Central Lancashire, Preston, UK; ${ }^{3}$ Faculty of Pharmacy, Medical University of Lodz, Lodz, Poland; ${ }^{4}$ School of Pharmacy and Biomedical Sciences, University of Central Lancashire, Preston, UK; ${ }^{5}$ Faculty of Pharmacy with the Laboratory Medicine Division, Medical University of Warsaw, Warsaw, Poland; ' ${ }^{6}$ School of Pharmacy, University of Bradford, Bradford; 'King's College London, London, UK
\end{abstract}

\subsection{6/archdischild-2019-esdppp.22}

Background Despite regulatory advances, lack of age-appropriate formulations (AAFs) remains a challenge in paediatric practice. 3D-printing of oral dosage forms (ODFs) offers potential for AAFs for children. Optimising drug release from 3D-printed ODFs is an important technological step. Despite the abundant use of polyethylene oxides (PEOs) and their extensive use as an excipient, there have been no previous reports of applying this thermoplastic polymer species alone to fused deposition modelling (FDM) 3D printing. We assessed the impact of polymer molecular weight $(\mathrm{MW})$ on the mechanical properties of the resultant filaments and their rheological properties. In the FDM 3D printing process, we also tested the effect of an innovative radiator-like design of the ODF on the acceleration of drug release patterns. Methods Blends of PEO (MW: 100K, 200K, 300K, 600K or $900 \mathrm{~K}$ ) with PEG 6K (plasticiser) and a model drug (theophylline) were prepared by hot-melt extrusion. The resultant filaments were used as a feed for a FDM 3D printer to fabricate innovative designs of ODFs in a radiator-like geometry with inter-connected paralleled plates and inter-plate spacing of either $0.5 \mathrm{~mm}, 1 \mathrm{~mm}, 1.5 \mathrm{~mm}$ or $2 \mathrm{~mm}$.

Results Varying blends of PEO and PEG allowed formation of mechanically resistant filaments (maximum load at break of $357,608,649,882,781 \mathrm{~N}$ for filament produced with $100 \mathrm{~K}$, $200 \mathrm{~K}, 300 \mathrm{~K}, 600 \mathrm{~K}$ or $900 \mathrm{~K}$, respectively). Filaments of PEO at a MW of $200 \mathrm{~K}-600 \mathrm{~K}$ were compatible with FDM 3D printing. Further increase in PEO MW resulted in elevated shear viscosity $\left(>10^{4} \mathrm{~Pa} . \mathrm{S}\right)$ at the printing temperature and hindered material flow during FDM $3 \mathrm{D}$ printing. A minimum spacing $(1 \mathrm{~mm})$ between parallel plates of the radiator-like design was essential to boost drug release from the structure. Conclusion These findings are essential in the development of next-generation personalised drug delivery doses using specialised polymer/polymer blends purposely optimised for FDM 3D printing. Disclosure(s) Nothing to disclose

\section{CAN CHILDREN SWALLOW TABLETS? OUTCOME DATA FROM A FEASIBILITY STUDY TO ASSESS THE SWALLOWABILITY AND ACCEPTABILITY OF DIFFERENT SIZED PLACEBO TABLETS IN CHILDREN AND YOUNG PEOPLE (CREATING ACCEPTABLE TABLETS - CAT)}

\footnotetext{
${ }^{1} \mathrm{~L}$ Bracken*, ${ }^{1} \mathrm{E}$ McDonough, ${ }^{2} \mathrm{~S}$ Ashleigh, ${ }^{2} \mathrm{~F}$ Wilson, ${ }^{2} \mathrm{U}$ Ohia, ${ }^{3} \mathrm{P}$ Mistry, ${ }^{4} \mathrm{H}$ Jones, ${ }^{4} \mathrm{~N}$ Kanji, ${ }^{5} \mathrm{~F}$ Liu, ${ }^{1} \mathrm{M}$ Peak. ${ }^{1}$ Paediatric Medicines Research Unit, Alder Hey Children's NHS Foundation Trust; ${ }^{2}$ NIHR Alder Hey Clinical Research Facility, Liverpool; ${ }^{3}$ Children's Cancer Trials Team, Cancer Research UK Clinical Trials Unit, Institute of Cancer and Genomic Sciences, University of Birmingham, Birmingham; ${ }^{4}$ Quotient Sciences, Nottingham; ${ }^{5}$ Department of Pharmacy, Pharmacology and Postgraduate Medicine, University of Hertfordshire, Hatfield, UK
}

Background It can be challenging to administer medicines to children and young people (CYP); due to the lack of available age-appropriate formulations. Developing medicines that are acceptable to CYP has the potential to improve treatment outcomes. ${ }^{1}$ Acceptability has been defined as 'an overall ability of the patient and caregiver (defined as 'user') to use a medicinal product as intended'. ${ }^{2}$ There is limited evidence for the acceptability of tablets in CYP. This feasibility study aimed to investigate the swallowability and acceptability of different sized placebo tablets in CYP aged 4-12.

Method Participants were asked to swallow three different sized placebo tablets; $6 \mathrm{~mm}, 8 \mathrm{~mm}$ and $10 \mathrm{~mm}$, smallest to largest. Both healthy children and NHS patients were recruited. The researcher observed and recorded children's facial expressions as they swallowed each tablet. ${ }^{3}$ Following administration, an internal inspection of the mouth was conducted to identify any residue or non-swallowed tablet. ${ }^{4}$ Participants completed a questionnaire about the acceptability of each tablet. For analysis participants were stratified by age: 4-8 and 9-12 years.

Results 55 participants were recruited to the study. 30 children were in the younger group, of which $23 \%$ had taken a tablet before. $84 \%$ of the 25 older children had previously taken a tablet. $100 \%$ of participants attempted to swallow the $6 \mathrm{~mm}$ tablet, with $67 \%$ of younger children and all older children successfully swallowing the tablet. All participants in the older group attempted to swallow the $8 \mathrm{~mm}$ and $10 \mathrm{~mm}$ tablet with $100 \%$ successfully swallowing the $8 \mathrm{~mm}$ and $96 \%$ successfully swallowing the $10 \mathrm{~mm}$ tablet. $77 \%$ of younger children attempted to swallow the $8 \mathrm{~mm}$ tablet, with $91 \%$ succeeding. $70 \%$ of younger children attempted the $10 \mathrm{~mm}$ tablet, with 95\% succeeding.

Conclusion This study demonstrates that tablets of $6 \mathrm{~mm}, 8 \mathrm{~mm}$ and $10 \mathrm{~mm}$ are potentially an acceptable formulation for children aged 4-12 years.

\section{REFERENCES}

1. Venables $\mathrm{R}$, Batchelor HK, Hodson J, Stirling $H$, Marriott J. Determination of formulation factors that affect oral medicines acceptability in a domiciliary paediatric population. Int J Pharm 2015;480(1-2):55-62.

2. Kozarewicz P. Regulatory perspectives on acceptability testing of dosage forms in children. Int J Pharm 2014;469:245-8. doi:10.1016/j.jpharm.2014.03.057. European Medicines Agency. Guideline on pharmaceutical development of medicines for paediatric use. 2013;44:1-24

3. Zeinstra GG, Koelen MA, Colindres $D$, et al. Facial expressions in school-aged children are a good indicator of 'dislikes'but not of 'likes'. Food Quality and Preference 2009 December 2009;20(8):620-624

4. Klingmann V, Spomer N, Lerch $C$, et al. Favorable Acceptance of Mini-Tablets Compared with Syrup: A Randomized Controlled Trial in Infants and Preschool Children. The Journal of Pediatrics 2013 December 2013;163(6):1728-1732.e1.

Disclosure(s) Nothing to disclose

\section{O24 IVERMECTIN IN CHILDREN: WHAT IS THE RIGHT DOSE TO ACHIEVE EQUIVALENT EXPOSURE COVERAGE IN CHILDREN AND ADULTS?}

\footnotetext{
${ }^{1,2,3} \mathrm{JM}$ Brussee ${ }^{*},{ }^{1,2} \mathrm{JD}$ Schulz, ${ }^{1,4,5} \mathrm{JT}$ Coulibaly, ${ }^{1,2}{ }^{2}$ Keiser, ${ }^{2,3,6} \mathrm{M}$ Pfister. ${ }^{1}$ Department of Medical Parasitology and Infection Biology, Swiss Tropical and Public Health Institute; ${ }^{2}$ University of Basel; ${ }^{3} U K B B$, Pediatric Pharmacology and Pharmacometrics, University Children's Hospital Basel, Basel, Switzerland; ${ }^{4}$ Unité de Formation et de Recherche Biosciences, Université Félix Houphouët-Boigny; ${ }^{5}$ Centre Suisse de Recherches Scientifiques en Côte d'Ivoire, Abidjan, Côte $d^{\prime} I v o i r e ; ~ '{ }^{6}$ Certara LP, Princeton, NJ, USA
} 
Background The broad-spectrum antiparasitic drug ivermectin is widely used in children, and its use in children weighing < $15 \mathrm{~kg}$ is off-label, as little data is available to inform the use of ivermectin in this young age group. Pediatric doses associated with consistent exposure across age are still unknown. Therefore, we aim to identify a dosing strategy for ivermectin treatment in both pre-school-aged children (2-5 years of age) and school-aged children (6-12 years of age) that achieves equivalent exposure coverage in children and adults.

Methods A population pharmacokinetic model for ivermectin was developed based on data collected in 80 pre-school-aged children (2-5 years), 120 school-aged children (6-12 years), ${ }^{1}$ and eleven adults, ${ }^{2}$ receiving an oral dose of $100-600 \mu \mathrm{g} / \mathrm{kg}$ ivermectin. Model-based simulations were performed to optimize pediatric dosing to achieve consistent exposure across various age groups.

Results Clearance per kilogram was higher in children than in adults, with a median (90\% confidence interval) of 0.35 $(0.12-0.73) \mathrm{L} / \mathrm{h} / \mathrm{kg}$ in children compared to $0.20(0.10-0.31)$ $\mathrm{L} / \mathrm{h} / \mathrm{kg}$ in adults. As a result, ivermectin exposure in children following a $200 \mu \mathrm{g} / \mathrm{kg}$ dose is $\sim 30 \%$ lower than in adults. An increased dose of 250 and $300 \mu \mathrm{g} / \mathrm{kg}$ would be needed in school-aged children (6-12 years) and pre-school-aged children (2-5 years), respectively, to achieve equivalent exposure coverage in children and adults. Alternatively, we propose a heightbased dosing schedule with a stepwise increase in number of administered 3-mg-tablets from 1 to 5 for children in subSaharan Africa with a height of 75-90 cm, 90-130 cm, 130$150 \mathrm{~cm}, 150-165 \mathrm{~cm}$, and $165-175 \mathrm{~cm}$.

Conclusion We report the first dosing strategy for the widelyused drug ivermectin that is associated with equivalent exposure coverage in children and adults. Further studies are necessary to establish the safety and efficacy of appropriate doses in the pediatric population.

\section{REFERENCES}

1. Wimmersberger D, Coulibaly JT, Schulz JD, Puchkow M, Huwyler J, N'Gbesso Y, et al. Efficacy and Safety of Ivermectin Against Trichuris trichiura in Preschoolaged and School-aged Children: A Randomized Controlled Dose-finding Trial. Clin Infect Dis. 2018; 67(8):1247-55.

2. Schulz JD, Neodo A, Coulibaly JT, Keiser J. Development and validation of a LCMS/MS method for ivermectin quantification in dried blood spots: application to a pharmacokinetic study in Trichuris trichiura-infected adults. Anal. Methods, 2018, 10, 2901-9.

Disclosure(s) JMB declares no potential conflicts of interest. JDS declares no potential conflicts of interest. JTC declares no potential conflicts of interest. JK was financially supported by the Bill and Melinda Gates Foundation (OPP1153928) and an ERC grant (CoG 614739 A-HERO), and declares no other potential conflicts of interest. MP has a part-time employment with the consulting company Certara LP (USA), and declares no other potential conflicts of interest.

\section{CLAVULANATE STABILITY IN CHILD-APPROPRIATE FORMULATIONS IS INADEQUATE FOR USE IN TREATING YOUNG CHILDREN IN ASIA}

${ }^{1}$ I Mack*, ${ }^{2} \mathrm{M}$ Sharland, ${ }^{3} \mathrm{~S}$ Rehm, ${ }^{3} \mathrm{~K}$ Rentsch, ${ }^{4} \mathrm{~J}$ Bielicki. 'University Children's Hospital Basel (UKBB), Basel, Switzerland; '2St George's University of London, London, UK; ${ }^{3}$ University Hospital of Basel; "4niversity Children's Hospital Basel (UKBB), Basel, Switzerland

10.1136/archdischild-2019-esdppp.25
Background Amoxicillin-clavulanate (AMC) is among the most frequently used antibiotic for paediatric infections globally. AMC child-appropriate formulations are largely limited to dry powder suspensions, which have to be stored refrigerated once reconstituted due to stability limitations of clavulanate.

Methods Oral Amoxicillin (AMX) and AMC formulations were identified from IQVIA-MIDAS wholesale data, and 2015 antibiotic consumption in courses/1000 child-years was estimated in Bangladesh, India, Indonesia, Pakistan, Philippines and Vietnam with an assumed average treatment of 7 days. Costs per course in US-\$ standardised to 2015 were estimated from the same dataset. Nationally representative data on access to a refrigerator was extracted from the Demographic \& Health Surveys Program. Degradation under different temperature conditions of two different AMC suspensions commercially available in Switzerland was tested. Average degradation (three bottles of each product) was measured during 8 days with ambient temperatures of $8^{\circ} \mathrm{C}$ versus $28^{\circ} \mathrm{C}$.

Results In India and Pakistan more AMC than AMX courses were sold. In all countries AMC was at least twice and up to 10 times as expensive as AMX. Access to refrigeration was below 45\%, even in countries with a high number of sold AMC courses (compared with AMX). In the evaluated co-formulated products, clavulanate showed a maximum degradation of $34 \%$ at $8^{\circ} \mathrm{C}$, and $73 \%$ at $28^{\circ} \mathrm{C}$ after 8 days. AMX was largely stable at $8^{\circ} \mathrm{C}$ but $13 \%$ degraded at $28^{\circ} \mathrm{C}$ after 8 days. Conclusions Oral amoxicillin-clavulanate suspensions are widely used in six Asian countries classified as middle-income countries by the World Bank. In reconstituted liquid AMC formulations, neither component is satisfactorily stable at room temperature. Storage conditions for stability are likely inadequate for AMC in many households in the six Asian countries of interest.

Disclosure(s) Nothing to disclose

\section{POPULATION PHARMACOKINETIC META-ANALYSIS OF INDIVIDUAL DATA TO DESIGN THE FIRST RANDOMIZED EFFICACY TRIAL OF VANCOMYCIN IN NEONATES AND YOUNG INFANTS}

${ }^{1} \mathrm{E}$ Jacqz-Aigrain, ${ }^{2} \mathrm{~S}$ Leroux, ${ }^{3} \mathrm{AH}$ Thomson, ${ }^{4} \mathrm{~K}$ Allegaert, ${ }^{5} \mathrm{EV}$ Capparelli, ${ }^{1} \mathrm{~V}$ Biran, ${ }^{6} \mathrm{~N}$ Simon, ${ }^{7} \mathrm{~B}$ Meibohm, ${ }^{8} \mathrm{Lo} \quad \mathrm{Y}-\mathrm{L},{ }^{9} \mathrm{R}$ Marqués, ${ }^{10} \mathrm{~J}-\mathrm{E}$ Peris, ${ }^{11} \mathrm{I}$ Lutsar, ${ }^{12} \mathrm{~J}$ Saito, ${ }^{12} \mathrm{H}$ Nayamura, ${ }^{13} \mathrm{JN}$ van den Anker, ${ }^{14} \mathrm{M}$ Sharland, ${ }^{15} \mathrm{~W}$ Zhao*. ${ }^{1}$ Hôpital Robert Debré, Paris; ${ }^{2} \mathrm{CHU}$ de Rennes, Rennes, France; ${ }^{3}$ University of Strathdyde, Glasgow, UK; ${ }^{4}$ Erasmus MC - Sophia Children's Hospital, Rotterdam, The Netherlands; ${ }^{5}$ University of California, San Diego, CA, USA; ${ }^{6}$ Hôpital de la Timone, Marseille, France; ${ }^{7}$ University of Tennessee Health Science Center, Memphis, TN, USA; ${ }^{8}$ University of Malaya, Malaysia, Malaysia; ${ }^{9} \mathrm{La}$ Fe Hospital; ${ }^{10}$ University of Valencia, Valencia, Spain; ${ }^{11}$ University of Tartu, Tartu, Estonia; ${ }^{12}$ National Children's Hospital National Center for Child Health and Development, Tokyo, Japan; ${ }^{13}$ University Children's Hospital Basel, Basel, Switzerland; ${ }^{14}$ St. George's Hospital, London, UK; ${ }^{15}$ Shandong University, Jinan, China

10.1136/archdischild-2019-esdppp.26

Background In the absence of consensus, the present metaanalysis was performed to determine an optimal dosing regimen of vancomycin for neonates.

Methods A 'meta-model' using NONMEM with 4894 concentrations from 1631 neonates was built and Monte Carlo simulations were performed to design an optimal intermittent infusion, aiming at reaching a target AUC0-24 of $400 \mathrm{mg}$ *h/L at steady state in at least $80 \%$ of neonates. 